\title{
Ectopic congenital bronchogenic cyst accompanied by infection appearing in the cervical region of an elderly female patient: A case report
}

\author{
ZHONGLONG LIU, ZHUOWEI TIAN, CHENPING ZHANG and YUE HE \\ Department of Oromaxillofacial Head and Neck Oncology, Ninth People's Hospital, College of Stomatology, \\ Shanghai Jiao Tong University School of Medicine, Shanghai 200011, P.R. China
}

Received May 10, 2014; Accepted August 18, 2015

DOI: $10.3892 / 01.2015 .4022$

\begin{abstract}
Bronchogenic cysts (BCs) are rare congenital cystic lesions arising as a development malformation in the trachea-bronchial system during the embryonic period. The cysts mostly occur in the mediastinum, with an extremely low morbidity rate in the oromaxillofacial-head and neck region. The age distribution of patients who present with BCs shows a significant predominance towards young individuals compared with the elderly. The current study reports the case a 70-year-old female who presented with a painful mass in the right cervical region. Pre-operative computed tomography (CT) and ultrasound showed a heterogeneous hypodense cystic lesion accompanied by infection. A mass of gaseous density was observed on repeat $\mathrm{CT}$ following antibiotic therapy. The pathological examination after complete resection revealed a $\mathrm{BC}$, which can also be easily misdiagnosed as common congenital cervical cysts, such as a branchial cleft cyst or a thyroglossal duct cyst, among others. There was no recurrence or any discomfort during the 34-month follow-up period.
\end{abstract}

\section{Introduction}

Bronchogenic cysts (BC) occurring in the head and neck region were first reported by Park and Buford (1) in 1955. The cysts usually appear in the mediastinum and chest of infants and children, but they seldom occur in adults, particularly in elderly individuals (2-4). The ratio of males to females diagnosed with congenital cysts is 4:1 (5). According to their

Correspondence to: Professor Yue He, Department of Oromaxillofacial Head and Neck Oncology, Ninth People's Hospital, College of Stomatology, Shanghai Jiao Tong University School of Medicine, 639 Zhi-zao-ju Road, Shanghai 200011, P.R. China

E-mail: yuehe@sjtu.edu.cn

Key words: bronchogenic cyst, congenital, infection, elderly female location, $\mathrm{BC}$ can be divided into intrathoracic or extrathoracic $\mathrm{BC}$. Based on this foundation, Maier (6) further classified $\mathrm{BC}$ into five groups: The carinal, paratracheal, hilar, paraesophageal and atypical groups. BC of the cervical region belongs to the atypical group. BCs are easily confused with third or fourth branchial cleft cysts, particularly when the lesions occur in the root of cervical region accompanied by deep neck abscesses $(2,3)$. Considering the recurrence of cysts following incomplete excision and the possibility of malignant biological behavior, a complete resection is the optimal method for the treatment of $\mathrm{BC}$. The current study reports the case a 70-year-old female who presented with a painful mass in the right cervical region, and also provides a review of the literature.

\section{Case report}

In August 2012, a 70-year-old female presented to the Ninth People's Hospital (College of Stomatology, Shanghai Jiao Tong University School of Medicine, Shanghai, China) with a painful mass in the right cervical region that had been apparent for one month. There had been no mass in the area prior to the appearance of the clinical symptoms. A physical examination found a soft, smooth, tender, unmovable cystic mass, $4.0 \mathrm{~cm}$ in diameter, below the right thyroid gland. No fistulae or scars on the skin were detected. Slight mobility was discovered during patient aspiration.

Ultrasound examination showed a 46x31-mm, mixed echoic cystic mass below the right thyroid gland tissue, with a clear border. Computed tomography (CT) scans confirmed the presence of a $3.3 \times 3.0-\mathrm{cm}$ hypodense cystic mass with regular margins located in the right cervical region. A left shift of the trachea was also observed due to compression by the cyst (Fig. 1A). The pre-operative diagnosis was of a branchial cleft cyst accompanied by infection. Antibiotics (cefoxitin sodium, injected at a dose of $2 \mathrm{~g} /$ day for 3 days) were applied prior to the surgery due to symptoms of inflammation. Repeat CT showed a $2.9 \times 2.8-\mathrm{cm}$ oval mass of gaseous density, with a $\mathrm{C}$ value of $-988 \mathrm{HU}$. Only a thin septum, without evident perforation between the mass and trachea, was observed by CT (Fig. 1B-C); this showed 

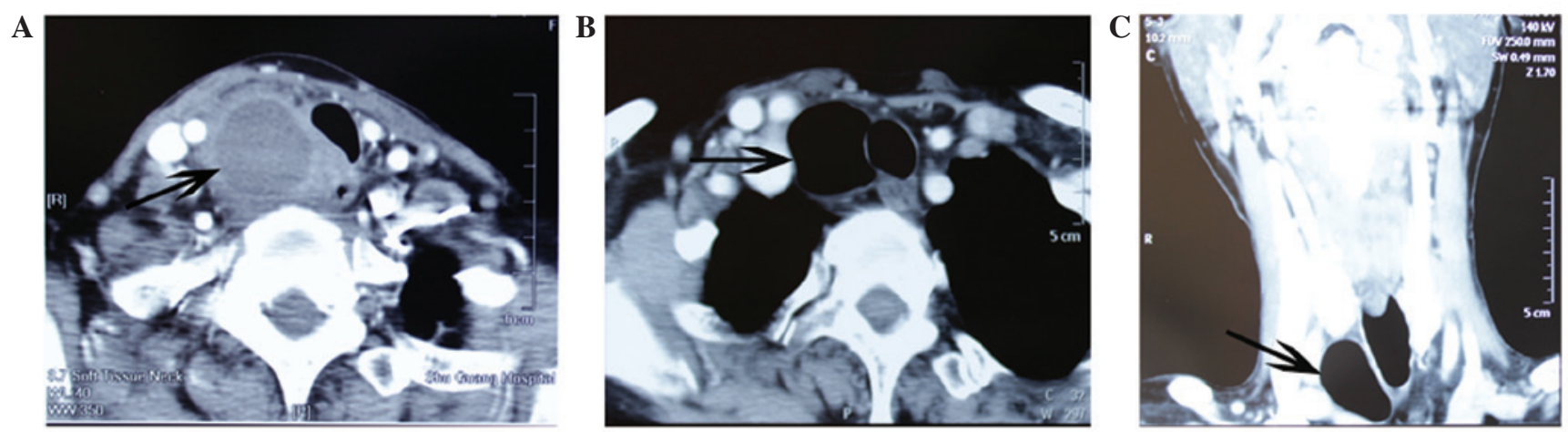

Figure 1. Computed tomography (CT) images of the bronchogenic cyst. (A) Axial CT showing hypodense image of the cyst prior to antibiotic therapy. Severe compression of the trachea by the cyst can be observed (arrow). (B) Axial CT showing a cyst of gaseous density (arrow) following antibiotic therapy. A papery septum without perforation can also be observed. (C) The coronal CT showed the location of the cyst, which had a distinct boundary with the right lobe of the thyroid (arrow).
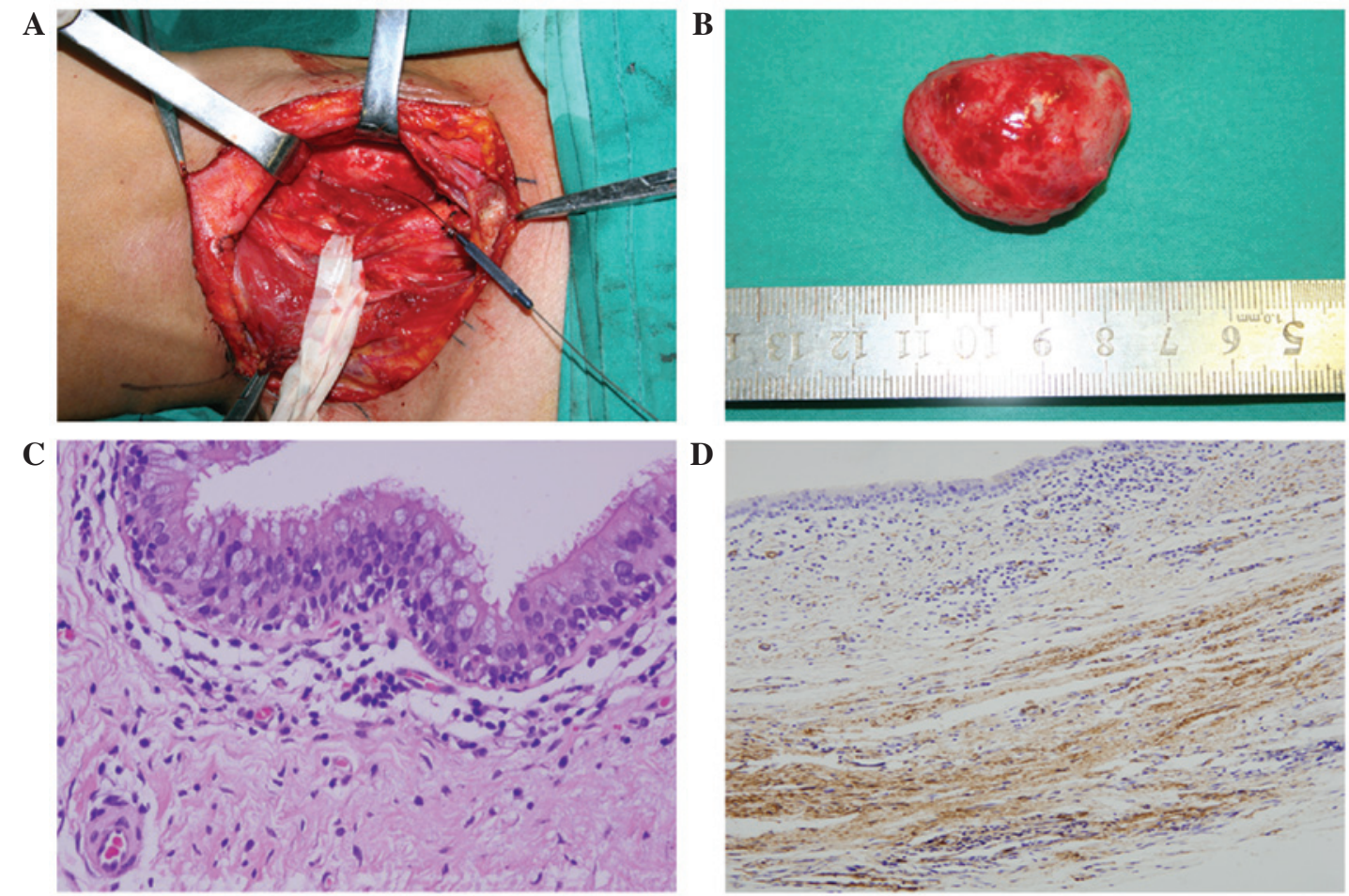

Figure 2. (A) Exposure of the cyst (arrow) following separation of the adjacent tissue during surgery. (B) The resected cyst, measuring $3.7 \mathrm{~cm}$ in diameter. The oval lesion was filled with gas. (C) The histopathology of the cyst wall showing the lining of pseudostratified ciliated columnar epithelium accompanied by scattered mucous metaplastic cells (hematoxylin and eosin staining; original magnification, x40). (D) Smooth muscle actin immunochemistry revealing a strongly positive reaction (arrow) in the cyst wall (original magnification, x20).

the elimination of inflammation and the real nature of the disease.

Surgery was performed when inflammation subsided after antibiotic treatment. A rounded mass filled with air was detected attached to the right trachea with a short fibrous duct during the surgery. After complete resection with clear margins, the mass was able to return to its original shape following extraction of the air content, which was performed using a 10-ml injector (Fig. 2A and B). The gross pathology revealed a unilocular cyst filled with gas. Microscopically, the lining of the cyst was pseudostratified cilliated columnar epithelium accompanied by scattered mucous metaplastic cells (Fig. 2C). Smooth muscle was found in the cyst wall, while cartilage was not observed (Fig. 2D). The histological diagnosis was of a $\mathrm{BC}$ accompanied by infection. The patient was followed up for 34 months, without experiencing any discomfort or recurrence.

\section{Discussion}

BCs are congenital cystic lesions arising from aberrated budding of the ventral foregut, which takes place during the gestation period (7). The deformity of the ventral foregut may occur in different phases of embryogenesis, with the result of the formation of $\mathrm{BC}$ in various positions. If the aberrated buddings arise in the initial stage of embryogenesis, it may 
lead to the $\mathrm{BC}$ being located along the tracheobronchial system, which differs from $\mathrm{BC}$ located in the mediastinum or lung parenchyma, due to malformations in development in the late stages $(8,9)$. BCs in the midline of the neck may have an intimate association with the development stage of the trachea during which the abnormal buddings occur, while cysts may be localized in the lower and lateral neck due to malformed development of the bronchial system (10).

BCs may present as asymptomatic cystic lesions in the head and neck region, with extremely low morbidity rates in elderly individuals, particularly elderly females (2-5). The size of the $\mathrm{BC}$ does not go unchanged and may enlarge with body growth. Disimilar to other studies, the case reported in the present study exhibited no evident mass or discomfort in the right neck during the 70 years prior to the onset of illness. It therefore remains uncertain as to how congenital disease occurs in elderly patients. Infection is not usually apparent in $\mathrm{BC}$ according to the cases previously reported in the literature (3). Cyst infections are usually caused by the communication of the cyst with the tracheal-bronchus system through fibrous connections, which may not be easily observed by $\mathrm{CT}$ or magnetic resonance imaging until during surgery (11). It has been reported that in adults, BCs of the thyroid and paratracheal region may be more likely to be infected compared with those in the supraclavicular and suprasternal notch (12). With regard to the present study, the patient suffered from local radioactive soreness or general infectious symptoms due to cyst infection. This infection could be easily observed in the third or fourth branchial cleft cysts, located in the root of the neck, and were characterized by local, recurrent deep abscesses.

Various types of image can be presented by CT examinations of $\mathrm{BC}$, including homogeneous density, fluid and air-liquid level masses. BC filled with air only, as observed in the present study, is extremely rare. Air-filled lesions may be observed on $\mathrm{CT}$ images when communication exists between the cyst and the tracheal-bronchus system (13). Bacteria can make best use of this narrow pathway to enter the cysts, which results in local or general infection, thus forming a deep abscess. In the present case, successive CT images are shown prior to and following anti-inflammatory therapy. In the inflammatory stage, the lesion presented with a heterogeneous hypodense cystic image, while after antibiotic treatment it became an image of a mass with gaseous density. There are no particular CT standards to distinguish $\mathrm{BCs}$ from other cysts.

BCs are characteristically lined by pseudostratified ciliated columnar epithelium, while squamous metaplasia, inflammation or totally necrotic tissues may be found when there has been previous infection $(14,15)$. The cyst wall may be comprised of cartilage, mucus-secreting goblet cells, fibrous connective tissue and smooth muscle, which may contribute to the cyst contraction, as observed in the present case. The majority of these components may easily be observed in intrathoracic $\mathrm{BC}$, but are rarely found in cervical $\mathrm{BC}$. In the analysis of cutaneous $\mathrm{BC}$, a previous study reported that smooth muscle was most likely to be found at a ratio of $70 \%$, followed by $57 \%$ glandular cells and $19 \%$ cartilage (16). It is really difficult to distinguish $\mathrm{BC}$ from branchial cleft cysts. Branchial cleft cysts usually appear in the upper triangle of the neck followed by the lateral neck, while BCs commonly occur in one side of the neck, with few cases reported in the upper cervical region (17). Therefore, the location of lesions cannot be used to form a definitive diagnosis. Branchial cleft cysts are typically lined with squamous epithelium, but scattered pseudostratified ciliated columnar epithelium may not be excluded. It is difficult to distinguish between BCs and branchial cleft cysts when relying solely on the histopathology. The clinical symptoms and histopathology manifestations should be additionally assessed to improve the distinction.

$\mathrm{BCs}$ are benign tumors in the majority of cases, although they may have an intimate connection with malignant tumors. When considering the potential risk of cyst recurrence and its complications, such as opportunistic infections and hemorrhage, a complete surgical resection of the $\mathrm{BC}$ should be recommended, particularly in elderly patients.

In conclusion, $\mathrm{BCs}$ are rare congenital lesions that seldom occur in the oromaxillofacial-head and neck region of elderly females, with few cases reported in the literature. The case described in the present study showed that congenital diseases, such as BC, may also appear in elderly individuals without clinical manifestations. It is a challenge for head and neck surgeons to form a correct differential diagnosis for $\mathrm{BC}$ and other cystic lesions, thus acquiring preferable therapeutic effects.

\section{Acknowledgements}

This study was supported by generous grants from the National Natural Science Foundation of China (no. 81271112), the Shanghai Municipal Human Resources and Social Security Bureau (no. 201312), SMC Rising Star (A) Scholar, supported by Shanghai Jiao Tong University.

\section{References}

1. Park $\mathrm{OK}$ and Buford $\mathrm{CH}$ : Bronchogenic cyst of neck and superior mediastinum. Ann Surg 142: 130-133, 1955.

2. Al-kasspooles MF, Alberico RA, Douglas WG, Litwin AM, Wiseman SM, Rigual NR, et al: Bronchogenic cyst presenting as a symptomatic neck mass in an adult: case report and review of the literature. Laryngoscope 114: 2214-2217, 2004.

3. Hazenberg AJ, Pullmann LM, Henke RP and Hoppe F: Recurrent neck abscess due to a bronchogenic cyst in an adult. J Laryngol Otol 124: 1325-1328, 2010.

4. Wilkinson N, Reid H and Hughes D: Intradural bronchogenic cysts. J Clin Pathol 45: 1032-1033, 1992.

5. Pul N and Pul M: Bronchogenic cyst of the scapular area in an infant: case report and review of the literature. J Am Acad Dermatol 31: 120-122, 1994.

6. Maier HC: Bronchiogenic cysts of the mediastinum. Ann Surg 127: 476-502, 1948.

7. Chapman KR and Rebuck AS: Spontaneous disappearance of a chronic mediastinal mass. Chest 87: 235-236, 1985.

8. Di Lorenzo M, Collin PP, Vaillancourt R and Duranceau A: Bronchogenic cysts. J Pediatr Surg 24: 988-991, 1989.

9. St-Georges R, Deslauriers J, Duranceau A, Vaillancourt R, Deschamps C, Beauchamp G, et al: Clinical spectrum of bronchogenic cysts of the mediastinum and lung in the adult. Ann Thorac Surg 52: 6-13, 1991.

10. Ustundag E, Iseri M, Keskin G, Yayla B and Muezzinoglu B: Cervical bronchogenic cysts in head and neck region. J Laryngol Otol 119: 419-423, 2005.

11. Kostopoulos G, Efstathiou A, Skordalaki A and Fessatidis I: Bronchogenic cyst infected by Salmonella enteritidis followed gastroenteritis. Eur J Cardiothorac Surg 21: 935-937, 2002. 
12. Moz U, Gamba P, Pignatelli U, D'Addazio G, Zorzi F, Fiaccavento $S$ and Milesi F: Bronchogenic cysts of the neck: a rare localization and review of the literature. Acta Otorhinolaryngol Ital 29: 36-40, 2009.

13. Touloukian RJ: Air filled bronchogenic cyst presenting as a cervical mass in the newborn. J Pediatr Surg 17: 311-312, 1982.

14. Newkirk KA, Tassler AB, Krowiak EJ and Deeb ZE: Bronchogenic cysts of the neck in adults. Ann Otol Rhinol Laryngol 113: 691-695, 2004.
15. Mehta RP, Faquin WC and Cunningham MJ: Cervical bronchogenic cysts: a consideration in the differential diagnosis of pediatric cervical cystic masses. Int J Pediatr Otorhinolaryngol 68: 563-568, 2004

16. Zvulunov A, Amichai B, Grunwald MH, Avinoach I and Halevy S: Cutaneous bronchogenic cyst: delineation of a poorly recognized lesion. Pediatr Dermatol 15: 277-281, 1998.

17. Shimazu R, Kuratomi Y and Inokuchi A: A case of an upper cervical bronchogenic cyst in an adult. Auris Nasus Larynx 33: 351-353, 2006. 